\title{
PHD Defence: “Consuming Communication: Promotion, Expertise, and Sustainability in an Age of Participatory Media" By Michael Felczak
}

\section{Sibo Chen}

School of Communication

Simon Fraser University

Our dear colleague, Michael Felczak, successfully defended his PHD dissertation on November 3, 2014. Here is the abstract of his dissertation "Consuming Communication: Promotion, Expertise, and Sustainability in an Age of Participatory Media":

\begin{abstract}
Taking as its point of departure the historical processes of mediatization and reflexive modernization, this dissertation examines the circuits of promotion, expertise, and meaning that mutually constitute the production and consumption of the smartphone. Through a focus on the online media practices of manufacturers, technology publishers, and consumers, the research maps the sociocultural mediation of this ubiquitous communication technology and considers the material aspects of this mediation in light of global environmental risk.
\end{abstract}

As privileged media producers, manufacturers signify smartphones as revolutionary achievements of technoscience: powerful artefacts that expand user capacity for agency, enjoyment, and sociability. Within a context of networked individualism, smartphones are positioned as shape-shifting devices that may be continually adapted to fluid tastes, social settings, and evolving life scripts. Although technology obsolescence and production are primary contributors to overall environmental impact, manufacturers shift attention to e-waste disposal and consumer responsibility.

In their role as observers of the consumer electronics industry, technology publishers expand manufacturer promotion in time and space. As technology experts, authors circulate a collective taste for novelty, distinction, and performance. While published articles may be understood as practical resources for consumers navigating a complex space of artefacts and promotion, this expertise excludes considerations of long-term ownership, repair, and the environmental aspects of technology consumption.

This circuit of promotion and expertise provides a foundation for consumer sociability that both enlivens and undermines the activities of manufacturers and industry experts. While avid consumers express collective enthusiasm for new technologies, they are cynical towards promotion as a practice, question the predominant smartphone ideal, and attempt to influence manufacturer design strategies. This popular discourse broadens the scope of cultural resources available to users, but simultaneously reproduces dominant consumption norms that legitimize rapid technology obsolescence.

These findings suggest that despite increased public sensitivity to global environmental risks, the production and consumption of new communication technologies represents a continuation of first modernity processes. The disconnection between general environmental risk awareness and its specific manifestation in everyday life points towards the need for an expanded popular expertise and green citizenship as a basis for democratic rationalization and governance of media technology. 
Chen

\section{Keywords}

Digital media; mobile technology; consumer culture; advertising and promotion; environment and sustainability; technology design 